\title{
Dietary patterns and survival in older Dutch women ${ }^{1-4}$
}

\author{
Patricia MCM Waijers, Marga C Ocké, Caroline TM van Rossum, Petra HM Peeters, Christina Bamia, \\ Yiannis Chloptsios, Yvonne T van der Schouw, Nadia Slimani, and H Bas Bueno-de-Mesquita
}

\begin{abstract}
Background: The need to gain insight into prevailing eating patterns and their health effects is evident.

Objective: This study aimed to identify dietary patterns and their relation to total mortality in older Dutch women.

Design: A principal component analysis of 22 food groups was used to identify dietary patterns in 5427 women aged 60-69 y who were included in the Dutch European Prospective Investigation into Cancer and Nutrition-Elderly cohort (follow-up: $\approx 8.2$ y). Mortality ratios for 3 major principal components were assessed by using Cox proportional hazard analysis.

Results: The most relevant principal components were a Mediterranean-like dietary pattern (high intakes of vegetable oils, pasta and rice, sauces, fish, and wine), a Traditional Dutch dinner dietary pattern (high intakes of meat, potatoes, vegetables, and alcoholic beverages), and a Healthy Traditional Dutch dietary pattern (healthy variant of the Traditional Dutch dinner dietary pattern; high intakes of vegetables, fruit, nonalcoholic drinks, dairy products, and potatoes). Differences in mean intakes between the highest and lowest tertiles of the 3 patterns were greatest for fruit, dairy products, potatoes, and alcoholic beverages. Consumption of Mediterranean foods, such as fish and oils, was relatively low overall. Two hundred seventy-seven deaths occurred in 44667 person-years. Independent of age, education, and other lifestyle factors, only the Healthy Traditional dietary pattern score was associated with a lower mortality rate. Women in the highest tertile of this pattern experienced a $30 \%$ reduction in mortality risk.

Conclusion: A Healthy Traditional Dutch diet, rather than a Mediterranean diet, appears beneficial for longevity and feasible for health promotion in older Dutch women. This diet is comparable with other reported healthy or prudent diets that have been shown to be protective against morbidity or mortality. Am J Clin Nutr 2006;83:1170-6.
\end{abstract}

KEY WORDS Dietary patterns, eating patterns, factor analysis, older Dutch women, European Prospective Investigation into Cancer and Nutrition-Elderly

\section{INTRODUCTION}

In Europe, chronic diseases, such as cardiovascular diseases and cancer, have become major causes of death (1). Because lifestyle factors, such as smoking, physical activity, and nutrition play important roles in the onset and progress of chronic diseases, they may be especially important in the elderly, who are at an increased risk of developing chronic diseases (2). Additionally, the acceleration in functional decline caused by external factors is generally believed to be reversible at any age (3). Because society is aging, the importance of a healthy diet in the elderly is evident. Reducing the burden of avoidable chronic diseases not only increases longevity and prolongs survival, but also improves quality of life by delaying or preventing chronic diseases. It is, therefore, of interest to identify existing dietary patterns in the elderly that are more or less healthy.

In the past decade, the analysis of dietary patterns, in addition to studying individual dietary components, has emerged as a method of exploring the relation between diet and disease. The individual component approach may uncover several important relations between dietary factors and disease; however, it passes over the complexity of the true relation between diet and disease. The major limitations of this approach were addressed by $\mathrm{Hu}(4)$ : intake of various dietary factors is highly correlated, many interactions between components of a diet and disease risk may exist, and it may, in practice, not be possible to detect small differences in disease risk from single nutrients. Studying dietary patterns in relation to health accounts for these issues and can additionally enhance our understanding of dietary practice and provide guidance for nutrition intervention and education.

\footnotetext{
${ }^{1}$ From the National Institute for Public Health and the Environment, Bilthoven, Netherlands (PMCMW, MCO, CTMvR, and HBBM); the Julius Center for Health Sciences and Primary Care, University Medical Center Utrecht, Netherlands (PHMP and YTvdS); the Department of Hygiene and Epidemiology, University of Athens Medical School, Athens, Greece (CB and $\mathrm{YC}$ ); and the International Agency for Research on Cancer, Lyon, France (NS).

${ }^{2}$ The authors are solely responsible for the publication and the publication does not represent the opinion of the European Community. The Community is not responsible for any use that might be made of data appearing in this work.

${ }^{3}$ Supported by the Quality of Life and Management of Living resources Program of the European Commission (DG Research, contract no. QLK6CT-2001-00241), coordinated by the Department of Hygiene and Epidemiology, University of Athens Medical School, Athens, Greece; the Europe against Cancer Program of the European Commission (DG SANCO) for the project EPIC, coordinated by the International Agency for Research on Cancer (World Health Organization); the Dutch Ministry of Public Health, Welfare and Sports (Netherlands); the Greek Ministry of Health and the Greek Ministry of Education (Greece); and a fellowship honoring Vasilios and Nafsika Tricha (Greece).

${ }^{4}$ Reprints not available. Address correspondence to P Waijers, Centre for Nutrition and Health, National Institute for Public Health and the Environment, PO Box 1, 3720 BA Bilthoven, Netherlands. E-mail: patricia.waijers@rivm.nl.

Received August 12, 2005

Accepted for publication January 18, 2006
} 
Recently, an extensive review of dietary pattern studies showed that many variations of a "healthy" or "prudent" dietary pattern, which were positively associated with better health outcome, have been identified across different populations (5). One of the main aims of the European Prospective Investigation into Cancer and Nutrition (EPIC)-Elderly project was also to study the relation between a posteriori dietary patterns and mortality. Within this project, a posteriori dietary patterns have been identified in elderly persons from 9 European countries. Two major principal components were detected: a vegetable-based diet and a sweet- and fat-dominated diet (6). Compared with southern European participants, Dutch (and most northern European) EPIC-Elderly participants scored slightly negative on the first component, whereas the Dutch were indifferent to the second principal component. Eating patterns may be culturally determined to a great extent. We therefore intended to identify dietary patterns in older Dutch women who participated in the EPICElderly study to find out the extent to which dietary patterns in this population of older women were similar to other reported patterns and to examine if existing dietary patterns are related to survival.

\section{SUBJECTS AND METHODS}

\section{Study population and follow-up}

The subjects were Dutch participants of the EPIC-Elderly study, a substudy of the EPIC study. EPIC is a multicenter cohort study under the coordination of the International Agency for Research on Cancer that examined the role of diet on the etiology of cancer and other chronic diseases. Details on the design and methods of the EPIC study have been described previously $(7,8)$. In brief, over half a million apparently healthy volunteers were recruited for the EPIC study from 10 European countries. Two Dutch cohorts, enrolled between 1993 and 1997, contribute to the EPIC study. The Dutch Prospect-EPIC cohort is composed of volunteers recruited from among women who were participating in a regional breast cancer screening program. The cohort is composed of 17357 women aged 50-69 y at enrollment who reside in Utrecht and its vicinity. The study population was described in detail elsewhere (9). The Monitoring Project on Risk Factors for Chronic Diseases in the Netherlands (MORGEN)EPIC cohort is composed of 22769 men and women aged 20-64 $\mathrm{y}$ from a random sample of the Dutch population from 3 towns in the Netherlands: Amsterdam, Doetinchem, and Maastricht. The study population was described in detail elsewhere (10). The Medical Ethics Committee of TNO (Netherlands Organization for Applied Scientific Research) approved the study, and the study was conducted according to the guidelines of the Helsinki declaration. Data on vital status, including dates of emigration or death up to 7 November 2002, were obtained through the National Population Database. The objectives of the EPIC-Elderly project were to investigate the prevailing dietary patterns in $\mathrm{Eu}-$ ropean elders (persons aged $\geq 60 \mathrm{y}$ at enrollment) and to study the role of these patterns on their health and longevity.

Of the initial 6729 Dutch men and women aged $\geq 60 \mathrm{y}$ at recruitment, all 314 men were excluded because their number was too small to consider separately. Vital status was ascertained for 6312 of the 6415 women. Of the women, 732 were excluded because they had self-reported myocardial infarction, stroke, cancer, or a combination of these at enrollment. Another 145 women were excluded due to missing information on one or more of the dietary, anthropometric, or lifestyle variables. Also, 8 participants who died within the first year after enrollment were excluded. The remaining 5427 participants (5194 from ProspectEPIC and 233 from MORGEN-EPIC) were included in the study. The mean follow-up period was $8.2 \mathrm{y}$

\section{Dietary intakes}

Information on foods and beverages consumed during the year preceding enrollment was collected with the use of a validated semiquantitative food-frequency questionnaire $(11,12)$. The questionnaire contained questions on habitual consumption frequency during the past year. The questionnaire enables estimation of the average daily consumption of 178 food items. The quantity consumed was estimated in commonly used units, household measures, or by colored photographs of foods shown in different portion sizes. Intake of each of the foods was calculated in grams per day, taking into account standard recipes. Total energy (in kJ/d) and ethanol (in g/d) intakes were estimated by using an extended version of the 1996 computerized Dutch food composition table (13).

Foods were classified into 17 main groups [according to a common classification (Table 1)] and into 124 subgroups, which were constructed for the EPIC study. This classification groups foods that could be described and quantified according to common rules across countries for the purpose of the EPIC calibration study, which used a 24-h diet recall (7). All main groups of this classification were considered in the present analysis, except for the miscellaneous category. In addition, a soy (soy and soy products) category was considered. However, some main groups were broken down: "cereals and cereal products" was divided into "pasta, rice, and other grain", "bread", and "other cereals and cereal products" categories; "added fats" was divided into "vegetable oils", "butter", and "margarine" categories; and "alcoholic beverages" was divided into "wine" and "other alcoholic beverages" categories - this resulted in 22 food groups that were used in the EPIC-Elderly study.

\section{Lifestyle and anthropometric variables}

Data on several lifestyle and health variables were recorded with the use of a core lifestyle questionnaire, which included questions on education, history of previous illnesses, history of smoking, and physical activity (occupational and leisure time). To calculate a score for daily physical activity during leisure time, the time spent on each of several activities (in h/wk) was multiplied by an energy cost coefficient to convert h/wk to kJ, and the kJs were summed.

Physical examinations were conducted by trained paramedics according to a standardized protocol. Weight (measured to the nearest $100 \mathrm{~g}$ on calibrated scales), height, and waist and hip circumferences (measured to the nearest $0.5 \mathrm{~cm}$ ) were measured while the participants were wearing only light indoor clothing and no shoes. Body mass index (BMI) was calculated as weight (in $\mathrm{kg}$ )/height ${ }^{2}$ (in $\mathrm{m}$ ).

\section{Statistical analysis}

For each of the 22 food groups (Table 1), a regression analysis was performed on the food group to control for energy intake. The residuals of these linear regressions were the variables entered in the factor analysis (14). 
TABLE 1

Factor loadings and consumption of each food group by tertile $(\mathrm{T})$ of the 3 major principal components in women of the Dutch European Prospective Investigation into Cancer and Nutrition-Elderly cohort ${ }^{l}$

\begin{tabular}{|c|c|c|c|c|c|c|c|c|c|c|c|c|}
\hline \multirow[b]{2}{*}{ Food groups } & \multicolumn{4}{|c|}{ Mediterranean-like dietary pattern } & \multicolumn{4}{|c|}{ Traditional Dutch dinner dietary pattern } & \multicolumn{4}{|c|}{ Healthy Traditional dietary pattern } \\
\hline & $\begin{array}{l}\text { Factor } \\
\text { loading }\end{array}$ & $\mathrm{T} 1$ & $\mathrm{~T} 2$ & $\mathrm{~T} 3$ & $\begin{array}{l}\text { Factor } \\
\text { loading }\end{array}$ & $\mathrm{T} 1$ & $\mathrm{~T} 2$ & $\mathrm{~T} 3$ & $\begin{array}{l}\text { Factor } \\
\text { loading }\end{array}$ & $\mathrm{T} 1$ & $\mathrm{~T} 2$ & $\mathrm{~T} 3$ \\
\hline & & $g / d$ & $g / d$ & $g / d$ & & $g / d$ & $g / d$ & $g / d$ & & $g / d$ & $g / d$ & $g / d$ \\
\hline Potatoes & $-0.30^{2}$ & $119 \pm 63^{3}$ & $87 \pm 48$ & $66 \pm 44$ & $0.23^{2}$ & $73 \pm 45$ & $89 \pm 50$ & $110 \pm 67$ & $0.22^{2}$ & $74 \pm 45$ & $90 \pm 52$ & $107 \pm 66$ \\
\hline Vegetables & $0.20^{2}$ & $123 \pm 44$ & $130 \pm 45$ & $156 \pm 59$ & $0.22^{2}$ & $119 \pm 47$ & $135 \pm 47$ & $155 \pm 55$ & $0.47^{2}$ & $107 \pm 40$ & $131 \pm 40$ & $171 \pm 53$ \\
\hline Legumes & 0.02 & $9 \pm 11$ & $9 \pm 9$ & $10 \pm 10$ & 0.11 & $8 \pm 8$ & $8 \pm 9$ & $11 \pm 12$ & $0.21^{2}$ & $7 \pm 7$ & $8 \pm 9$ & $12 \pm 12$ \\
\hline Fruit & 0.19 & $205 \pm 115$ & $246 \pm 130$ & $294 \pm 165$ & -0.17 & $281 \pm 163$ & $246 \pm 127$ & $217 \pm 128$ & $0.35^{2}$ & $188 \pm 107$ & $240 \pm 116$ & $317 \pm 167$ \\
\hline Dairy products & 0.08 & $417 \pm 226$ & $451 \pm 234$ & $487 \pm 275$ & $-0.20^{2}$ & $517 \pm 265$ & $454 \pm 233$ & $384 \pm 224$ & $0.29^{2}$ & $371 \pm 213$ & $441 \pm 214$ & $543 \pm 279$ \\
\hline Pasta, rice, and other grain & $0.26^{2}$ & $14 \pm 14$ & $21 \pm 19$ & $35 \pm 39$ & 0.05 & $21 \pm 27$ & $22 \pm 24$ & $25 \pm 32$ & -0.16 & $2 \pm 37$ & $22 \pm 24$ & $18 \pm 19$ \\
\hline Bread & $-0.28^{2}$ & $125 \pm 43$ & $105 \pm 38$ & $90 \pm 39$ & -0.07 & $110 \pm 44$ & $107 \pm 42$ & $103 \pm 41$ & 0.13 & $99 \pm 43$ & $108 \pm 40$ & $113 \pm 43$ \\
\hline Other cereals & $0.28^{2}$ & $7 \pm 8$ & $11 \pm 10$ & $18 \pm 16$ & -0.16 & $15 \pm 15$ & $12 \pm 12$ & $10 \pm 10$ & -0.14 & $15 \pm 15$ & $12 \pm 11$ & $10 \pm 10$ \\
\hline Meat and meat products & -0.15 & $9 \pm 43$ & $85 \pm 41$ & $75 \pm 44$ & $0.51^{2}$ & $53 \pm 31$ & $83 \pm 34$ & $116 \pm 40$ & -0.01 & $84 \pm 42$ & $84 \pm 42$ & $84 \pm 46$ \\
\hline Fish and shellfish & $0.22^{2}$ & $7 \pm 7$ & $10 \pm 8$ & $15 \pm 15$ & 0.12 & $8 \pm 8$ & $11 \pm 11$ & $12 \pm 13$ & 0.01 & $11 \pm 11$ & $10 \pm 11$ & $11 \pm 12$ \\
\hline Eggs & 0.00 & $15 \pm 12$ & $16 \pm 13$ & $15 \pm 12$ & $0.21^{2}$ & $12 \pm 9$ & $15 \pm 11$ & $20 \pm 16$ & -0.04 & $16 \pm 14$ & $15 \pm 12$ & $15 \pm 12$ \\
\hline Vegetable oils & $0.35^{2}$ & $1 \pm 2$ & $2 \pm 3$ & $5 \pm 4$ & 0.15 & $2 \pm 3$ & $2 \pm 3$ & $4 \pm 4$ & 0.11 & $2 \pm 3$ & $2 \pm 3$ & $3 \pm 4$ \\
\hline Butter & -0.01 & $5 \pm 5$ & $5 \pm 6$ & $5 \pm 6$ & 0.05 & $4 \pm 6$ & $4 \pm 5$ & $5 \pm 6$ & $-0.32^{2}$ & $7 \pm 8$ & $4 \pm 4$ & $3 \pm 3$ \\
\hline Margarine & $-0.39^{2}$ & $21 \pm 10$ & $13 \pm 8$ & $9 \pm 7$ & 0.08 & $13 \pm 10$ & $14 \pm 10$ & $15 \pm 11$ & 0.09 & $13 \pm 10$ & $15 \pm 10$ & $15 \pm 11$ \\
\hline Sugar and confectionary products & -0.18 & $44 \pm 28$ & $35 \pm 22$ & $30 \pm 20$ & $-0.33^{2}$ & $48 \pm 27$ & $36 \pm 21$ & $26 \pm 19$ & $-0.23^{2}$ & $44 \pm 29$ & $36 \pm 22$ & $29 \pm 19$ \\
\hline Cakes and biscuits & -0.05 & $37 \pm 23$ & $36 \pm 22$ & $34 \pm 23$ & $-0.36^{2}$ & $47 \pm 26$ & $35 \pm 18$ & $25 \pm 17$ & -0.03 & $36 \pm 24$ & $37 \pm 22$ & $34 \pm 21$ \\
\hline Nonalcoholic beverages & 0.13 & $1316 \pm 387$ & $1398 \pm 419$ & $1519 \pm 512$ & 0.00 & $1406 \pm 448$ & $1410 \pm 434$ & $1417 \pm 468$ & $0.28^{2}$ & $1258 \pm 387$ & $1380 \pm 387$ & $1594 \pm 501$ \\
\hline Wine & $0.25^{2}$ & $11 \pm 32$ & $25 \pm 48$ & $63 \pm 101$ & 0.19 & $18 \pm 39$ & $26 \pm 47$ & $54 \pm 103$ & $-0.24^{2}$ & $55 \pm 101$ & $25 \pm 51$ & $17 \pm 37$ \\
\hline Other alcoholic beverages & 0.11 & $16 \pm 44$ & $27 \pm 60$ & $42 \pm 90$ & $0.26^{2}$ & $12 \pm 27$ & $20 \pm 39$ & $52 \pm 104$ & $-0.30^{2}$ & $53 \pm 103$ & $20 \pm 38$ & $11 \pm 28$ \\
\hline Sauces & $0.26^{2}$ & $6 \pm 6$ & $9 \pm 8$ & $14 \pm 16$ & 0.06 & $9 \pm 13$ & $10 \pm 10$ & $11 \pm 11$ & -0.07 & $11 \pm 15$ & $9 \pm 10$ & $9 \pm 9$ \\
\hline Soups & 0.03 & $65 \pm 68$ & $70 \pm 75$ & $72 \pm 78$ & 0.10 & $60 \pm 63$ & $67 \pm 69$ & $80 \pm 86$ & 0.08 & $59 \pm 60$ & $71 \pm 75$ & $77 \pm 83$ \\
\hline Soy products & $0.23^{2}$ & $1 \pm 2$ & $1 \pm 3$ & $4 \pm 8$ & $-0.28^{2}$ & $4 \pm 8$ & $1 \pm 2$ & $0 \pm 2$ & 0.08 & $1 \pm 5$ & $2 \pm 5$ & $2 \pm 6$ \\
\hline Eigenvalue & 2.24 & & & & 1.82 & & & & 1.48 & & & \\
\hline Variance explained (\%) & 10.2 & & & & 8.3 & & & & 6.7 & & & \\
\hline
\end{tabular}

${ }^{1} n=5427$.

${ }^{2}$ Absolute value $\geq 20$; contributed significantly to the dietary pattern.

${ }^{3} \bar{x} \pm \mathrm{SD}$ (all such values).

Dietary patterns were identified by means of a Principal Component Analysis (15). Principal Components were extracted by using the correlation matrix to adjust for unequal variances of the original variables. To identify the number of principal components to be retained, we used the criterion of eigenvalues exceeding 1, a scree plot, and the interpretability of each component. Food groups (residuals) with absolute scoring coefficients (or factor loadings) $>0.2$ were considered important contributors to a component. We labeled patterns based on the combinations of food groups that contributed most strongly to the respective component. Each person received a score for each identified pattern by summing the standardized values of the food groups (ie, the residuals) weighted by their factor loadings.

The association between dietary pattern and mortality was analyzed with Cox proportional hazards regression. For the 3 dietary components, Cox models were developed to control for age (continuous), BMI (continuous), the waist-to-hip ratio (continuous), total energy intake (continuous), physical activity at work (categorical: unemployed, sedentary occupation, standing occupation, manual work, or heavy manual work), physical activity during leisure time (in tertiles), smoking status (categorical: never, past use, $\leq 1$ cigarette/d, 2-10 cigarettes/d, 11-20 cigarettes/d, or $>20$ cigarettes/d), self-reported diabetes mellitus at enrollment, and educational achievement (categorical: none or primary school completed, technical or vocational school completed, secondary school completed, or higher degree). Mean intake of nutrients was calculated for tertiles of the relevant principal components. Tests for trend, Mantel-Haenszel chisquare test, and logistic regression were carried out for demographic and lifestyle characteristics and mean nutrient intakes according to tertiles of the principal components. SAS version 9.1 (SAS Institute, Cary, NC) statistical software package was used to perform the calculations.

\section{RESULTS}

The first 3 principal components were judged most relevant considering their interpretability and the scree plot. These 3 components explained $25 \%$ of the total variance in the consumption of the 22 food groups. The factor loadings for the first 3 principal components for the total population of older Dutch women are shown in Table 1, as well as the mean intakes of the 22 food groups for each tertile of a component.

The first principal component, which we labeled a Mediterranean-like dietary pattern, was characterized by higher loadings of pasta and rice, sauces, fish, and vegetables in combination with vegetable oils, wine, and other cereals. Foods often consumed in the Netherlands, such as potatoes, bread, and margarine, contributed negatively to this component. The second principal component, the Traditional Dutch dinner dietary pattern, had positive scorings for meat, potatoes, vegetables, eggs, and alcoholic beverages. It was low in intakes of dairy products, sweets, and pastries. The traditional Dutch dinner consists of a combination of potatoes, meat, and vegetables. The third component, which represented a Healthy (variant of the) Traditional Dutch diet, was high in intakes of vegetables, fruit, dairy products, potatoes, and legumes, and also nonalcoholic beverages. It was low in intakes of butter and alcoholic beverages.

Differences in the mean intakes of food groups between the highest and lowest tertiles of the 3 patterns were largest for 
TABLE 2

Nutrient intakes by tertile (T) of the 3 major principal components in women of the Dutch European Prospective Investigation into Cancer and NutritionElderly cohort ${ }^{1}$

\begin{tabular}{|c|c|c|c|c|c|c|c|c|c|c|c|c|}
\hline & \multicolumn{3}{|c|}{ Mediterranean-like dietary pattern } & \multirow[b]{2}{*}{$\begin{array}{l}P \text { for } \\
\text { trend }\end{array}$} & \multicolumn{3}{|c|}{ Traditional Dutch dinner dietary pattern } & \multirow[b]{2}{*}{$\begin{array}{l}P \text { for } \\
\text { trend }\end{array}$} & \multicolumn{3}{|c|}{ Healthy Traditional dietary pattern } & \multirow[b]{2}{*}{$\begin{array}{l}P \text { for } \\
\text { trend }\end{array}$} \\
\hline & $\mathrm{T} 1$ & $\mathrm{~T} 2$ & $\mathrm{~T} 3$ & & $\mathrm{~T} 1$ & $\mathrm{~T} 2$ & $\mathrm{~T} 3$ & & $\mathrm{~T} 1$ & $\mathrm{~T} 2$ & $\mathrm{~T} 3$ & \\
\hline Energy (kcal) & $1765 \pm 385^{2}$ & $1690 \pm 367$ & $1757 \pm 384$ & 0.54 & $1758 \pm 385$ & $1696 \pm 372$ & $1757 \pm 380$ & 0.96 & $1750 \pm 396$ & $1708 \pm 366$ & $1753 \pm 377$ & 0.78 \\
\hline $\begin{array}{l}\text { Protein (\% of } \\
\text { energy) }\end{array}$ & $15.9 \pm 2.3$ & $16.5 \pm 2.4$ & $16.2 \pm 2.5$ & 0.0038 & $15.4 \pm 2.2$ & $16.4 \pm 2.3$ & $16.9 \pm 2.6$ & $<0.0001$ & $15.1 \pm 2.2$ & $16.3 \pm 2.2$ & $17.3 \pm 2.4$ & $<0.0001$ \\
\hline $\begin{array}{l}\text { Carbohydrates (\% of } \\
\text { energy) }\end{array}$ & $46.0 \pm 5.9$ & $45.6 \pm 6.1$ & $45.2 \pm 6.6$ & $<0.0001$ & $49.7 \pm 5.2$ & $46.0 \pm 4.7$ & $41.2 \pm 5.5$ & $<0.0001$ & $43.7 \pm 6.6$ & $45.8 \pm 5.7$ & $47.4 \pm 5.8$ & $<0.0001$ \\
\hline Fat (\% of energy) & $36.7 \pm 5.3$ & $35.1 \pm 5.2$ & $33.8 \pm 5.4$ & $<0.0001$ & $33.5 \pm 5.0$ & $35.2 \pm 5.0$ & $36.9 \pm 5.7$ & $<0.0001$ & $36.2 \pm 5.5$ & $35.5 \pm 5.1$ & $33.9 \pm 5.4$ & $<0.0001$ \\
\hline $\begin{array}{l}\text { Saturated fatty acids } \\
\text { (\% of energy) }\end{array}$ & $16.3 \pm 2.8$ & $15.4 \pm 2.6$ & $14.2 \pm 2.7$ & $<0.0001$ & $15.1 \pm 2.8$ & $15.3 \pm 2.7$ & $15.5 \pm 3.0$ & $<0.0001$ & $15.9 \pm 3.1$ & $15.4 \pm 2.6$ & $14.5 \pm 2.6$ & $<0.0001$ \\
\hline $\begin{array}{l}\text { Monounsaturated } \\
\text { fatty acids ( } \% \text { of } \\
\text { energy) }\end{array}$ & $13.2 \pm 2.3$ & $12.8 \pm 2.3$ & $12.5 \pm 2.4$ & $<0.0001$ & $11.9 \pm 2.2$ & $12.9 \pm 2.1$ & $13.8 \pm 2.4$ & $<0.0001$ & $13.4 \pm 2.4$ & $13.0 \pm 2.2$ & $12.2 \pm 2.3$ & $<0.0001$ \\
\hline $\begin{array}{l}\text { Polyunsaturated fatty } \\
\text { acids ( } \% \text { of } \\
\text { energy) }\end{array}$ & $6.8 \pm 1.9$ & $6.5 \pm 1.8$ & $6.8 \pm 1.9$ & 0.45 & $6.2 \pm 1.8$ & $6.7 \pm 1.8$ & $7.2 \pm 2.0$ & $<0.0001$ & $6.5 \pm 1.8$ & $6.8 \pm 1.8$ & $6.9 \pm 2.0$ & $<0.0001$ \\
\hline Cholesterol (mg) & $203 \pm 70$ & $198 \pm 70$ & $194 \pm 69$ & $<0.0001$ & $174 \pm 61$ & $193 \pm 59$ & $229 \pm 76$ & $<0.0001$ & $207 \pm 74$ & $195 \pm 67$ & $194 \pm 67$ & $<0.0001$ \\
\hline Alcohol (g) & $3 \pm 7$ & $7 \pm 10$ & $12 \pm 15$ & $<0.0001$ & $4 \pm 6$ & $6 \pm 8$ & $13 \pm 16$ & $<0.0001$ & $13 \pm 15$ & $6 \pm 9$ & $4 \pm 7$ & $<0.0001$ \\
\hline Fiber (g) & $23 \pm 5$ & $22 \pm 5$ & $23 \pm 6$ & 0.82 & $23 \pm 6$ & $22 \pm 5$ & $23 \pm 6$ & 0.18 & $20 \pm 5$ & $22 \pm 4$ & $26 \pm 5$ & $<0.0001$ \\
\hline
\end{tabular}

$$
\begin{aligned}
& { }^{I} n=5427 . \\
& { }^{2} \bar{x} \pm \mathrm{SD} \text { (all such values). }
\end{aligned}
$$

intakes of fruit, dairy products, potatoes, wine, and other alcoholic beverages. Consumption of fish, vegetable oils, legumes, and soy products were relatively low in all tertiles of each factor.

Mean nutrient intakes for tertiles of each of the 3 principal components are presented in Table 2. Across tertiles of the Mediterranean-like dietary pattern, substantial decreases were seen for intakes of fat, particularly for saturated and monounsaturated fatty acids, and cholesterol. Alcohol intake increased from the first to the third tertile.

Protein and fat intakes, especially intake of monounsaturated fatty acids, increased, whereas intake of carbohydrates decreased, with increasing scores for the Traditional Dutch dinner dietary pattern. Cholesterol and alcohol intakes were also significantly higher for persons in the higher tertiles of this dietary pattern. In contrast, for persons that scored higher on the Healthy Traditional pattern, fat, cholesterol, and alcohol intakes were lower, whereas intakes of fiber, carbohydrates, and protein were higher.

The women were aged between 60 and $70 \mathrm{y}$ at baseline; $42 \%$ were aged $\geq 65$. One-third had no education or had only completed primary school education. Furthermore, almost one-half of the population had never smoked, $60 \%$ had a BMI $>25$, and $5 \%$ had a waist-to-hip ratio $>0.90$.

Percentages of the categorical sociodemographic and lifestyle variables for each tertile of the principal component scores are shown in Table 3. Women who scored high on the Mediterranean-like dietary pattern were younger, higher educated, and more often former smokers than were the women who had a low score on this pattern. Higher scores for the Traditional Dutch dinner dietary pattern were associated with women who had a lower level of education, were more current smokers, and were more overweight. Women that were less educated, more likely nonsmokers, had higher BMIs, and were more physically active scored higher on the Healthy Traditional dietary pattern.

During the follow-up of this cohort, 277 deaths occurred in 44667 person-years (mean follow-up length: $8.2 \mathrm{y}$ ). The association between the tertiles of the 3 principal components and overall mortality are shown in Figure 1. Independent of age, education, and other lifestyle factors, only the Healthy Traditional pattern score was significantly associated with a lower mortality rate. Women in the highest tertile of this pattern had a $30 \%$ lower mortality risk than those in lowest tertile $(95 \%$ CI for the hazard ratio: $0.52,0.95$ ). The Mediterranean-like dietary pattern showed a tendency to a reduced overall mortality. Mortality risk was higher in women in the highest tertile than those in the lower tertiles of the Traditional Dutch dinner dietary pattern; however, these hazard ratios were not statistically significant.

\section{DISCUSSION}

In this population of older Dutch women, we identified 3 major principal components: a Mediterranean-like dietary pattern, a Traditional Dutch dinner dietary pattern, and a Healthy Traditional dietary pattern. We did not observe a significant association between the Mediterranean-like or the Traditional Dutch dinner dietary patterns and mortality; however, mortality risk was reduced by $30 \%$ for women in the highest compared with the lowest tertile of the Healthy Traditional dietary pattern.

A limitation of the present study is that the 22 food groups entered into the analyses may not optimally represent dietary choices of Dutch persons. For example, as can be seen from Table 1 , soy products are only marginally consumed and yet represent a distinct food group. In contrast, it would have been appropriate to separate the "bread" category into white bread and brown bread, because Dutch people consume high quantities of several kinds of bread. The construction of and choice for these 22 food groups was made for the European EPIC-Elderly study, of which this cohort was a subcohort. These food groups were created to discern prevailing dietary patterns for a nonspecific European population; eating habits differ considerably between the various countries included in EPIC and these food groups may not be optimal to ascertain the dietary patterns in a specific country, such as the Netherlands. However, we decided to maintain these food groups to enable comparison. Our study population consisted of women aged between 60 and $70 \mathrm{y}$ at baseline. This group 
TABLE 3

Demographic and lifestyle characteristics by tertile (T) of the 3 major principal components in women of the Dutch European Prospective Investigation into Cancer and Nutrition-Elderly cohort ${ }^{l}$

\begin{tabular}{|c|c|c|c|c|c|c|c|c|c|c|c|c|c|c|c|}
\hline & \multicolumn{3}{|c|}{$\begin{array}{c}\text { Mediterranean- } \\
\text { like dietary } \\
\text { pattern }\end{array}$} & \multirow[b]{2}{*}{$\begin{array}{c}\text { Chi- } \\
\text { square } P\end{array}$} & \multirow[b]{2}{*}{$\begin{array}{l}P \text { for } \\
\text { trend }^{2}\end{array}$} & \multicolumn{3}{|c|}{$\begin{array}{c}\text { Traditional } \\
\text { Dutch dinner } \\
\text { dietary } \\
\text { pattern }\end{array}$} & \multirow[b]{2}{*}{$\begin{array}{c}\text { Chi- } \\
\text { square } P\end{array}$} & \multirow[b]{2}{*}{$\begin{array}{l}P \text { for } \\
\text { trend }^{2}\end{array}$} & \multicolumn{3}{|c|}{$\begin{array}{l}\text { Healthy } \\
\text { Traditional } \\
\text { dietary } \\
\text { pattern }\end{array}$} & \multirow[b]{2}{*}{$\begin{array}{c}\text { Chi- } \\
\text { square } P\end{array}$} & \multirow[b]{2}{*}{$\begin{array}{l}P \text { for } \\
\text { trend }^{2}\end{array}$} \\
\hline & $\mathrm{T} 1$ & $\mathrm{~T} 2$ & $\mathrm{~T} 3$ & & & $\mathrm{~T} 1$ & $\mathrm{~T} 2$ & $\mathrm{~T} 3$ & & & $\mathrm{~T} 1$ & $\mathrm{~T} 2$ & $\mathrm{~T} 3$ & & \\
\hline & & $\%$ & & & & & $\%$ & & & & & $\%$ & & & \\
\hline Age & & & & $<0.0001$ & & & & & 0.014 & & & & & 0.059 & \\
\hline $60-64$ y & 56 & 57 & 63 & & & 57 & 58 & 61 & & & 60 & 58 & 57 & & \\
\hline $65-70 y$ & 44 & 43 & 37 & & $<0.0001$ & 43 & 42 & 39 & & 0.0139 & 40 & 42 & 43 & & \\
\hline Education & & & & $<0.0001$ & & & & & 0.0002 & & & & & $<0.0001$ & \\
\hline None or primary school & 49 & 32 & 19 & & $<0.0001$ & 31 & 33 & 36 & & 0.0031 & 29 & 33 & 39 & & $<0.0001$ \\
\hline Technical school & 26 & 29 & 22 & & 0.0060 & 25 & 28 & 24 & & 0.37 & 22 & 28 & 27 & & 0.0005 \\
\hline Secondary school & 20 & 30 & 39 & & $<0.0001$ & 29 & 30 & 31 & & 0.40 & 36 & 29 & 25 & & $<0.0001$ \\
\hline University degree & 4 & 8 & 19 & & $<0.0001$ & 14 & 9 & 9 & & $<0.0001$ & 13 & 10 & 10 & & 0.0007 \\
\hline Smoking status & & & & 0.0002 & & & & & $<0.0001$ & & & & & $<0.0001$ & \\
\hline Never & 56 & 51 & 42 & & $<0.0001$ & 56 & 54 & 39 & & $<0.0001$ & 42 & 51 & 56 & & $<0.0001$ \\
\hline Former & 25 & 33 & 42 & & $<0.0001$ & 32 & 32 & 36 & & 0.023 & 35 & 34 & 31 & & 0.0039 \\
\hline Current & 19 & 16 & 15 & & 0.0010 & 12 & 14 & 25 & & $<0.0001$ & 23 & 15 & 13 & & $<0.0001$ \\
\hline BMI & & & & $<0.0001$ & & & & & $<0.0001$ & & & & & $<0.0001$ & \\
\hline$\leq 25 \mathrm{~kg} / \mathrm{m}^{2}$ & 35 & 37 & 42 & & & 46 & 38 & 30 & & & 44 & 38 & 32 & & \\
\hline$>25 \mathrm{~kg} / \mathrm{m}^{2}$ & 65 & 63 & 58 & & $<0.0001$ & 54 & 62 & 70 & & $<0.0001$ & 56 & 62 & 68 & & $<0.0001$ \\
\hline Waist-to-hip ratio & & & & 0.0050 & & & & & $<0.0001$ & & & & & 0.89 & \\
\hline$\leq 0.90$ & 92 & 93 & 95 & & & 95 & 93 & 92 & & & 94 & 93 & 94 & & \\
\hline$>0.90$ & 8 & 7 & 5 & & 0.0054 & 5 & 7 & 8 & & $<0.0001$ & 6 & 7 & 6 & & \\
\hline Physical activity at work & & & & 0.035 & & & & & 0.060 & & & & & $<0.0001$ & \\
\hline Sedentary & 81 & 80 & 81 & & 0.77 & 79 & 81 & 83 & & & 82 & 80 & 79 & & 0.0321 \\
\hline Standing & 9 & 10 & 11 & & 0.050 & 11 & 9 & 9 & & & 11 & 10 & 9 & & 0.084 \\
\hline Manual work & 6 & 7 & 6 & & 0.83 & 6 & 7 & 5 & & & 5 & 6 & 6 & & 0.15 \\
\hline Heavy manual work & 5 & 4 & 2 & & 0.0001 & 4 & 3 & 4 & & & 2 & 3 & 5 & & $<0.0001$ \\
\hline $\begin{array}{l}\text { Physical activity during } \\
\text { leisure time }\end{array}$ & & & & 0.70 & & & & & 0.62 & & & & & $<0.0001$ & \\
\hline Low & 34 & 36 & 34 & & & 35 & 34 & 34 & & & 39 & 32 & 33 & & $<0.0001$ \\
\hline Moderate & 34 & 35 & 35 & & & 34 & 36 & 34 & & & 33 & 37 & 34 & & 0.65 \\
\hline High & 32 & 30 & 31 & & & 31 & 30 & 31 & & & 28 & 31 & 34 & & $<0.0001$ \\
\hline
\end{tabular}

${ }^{1} n=5427$.

${ }^{2} P$ for trends are only shown if the Mantel Haenszel chi-square test was significant at the 0.05 level.

can be described as relatively young elderly women and, therefore, selection bias due to selective survival will not play an important role. However, the number of deaths was limited $(n=$ 277) because the women have only been followed for an average of $8.2 \mathrm{y}$. For this reason, only associations with total mortality

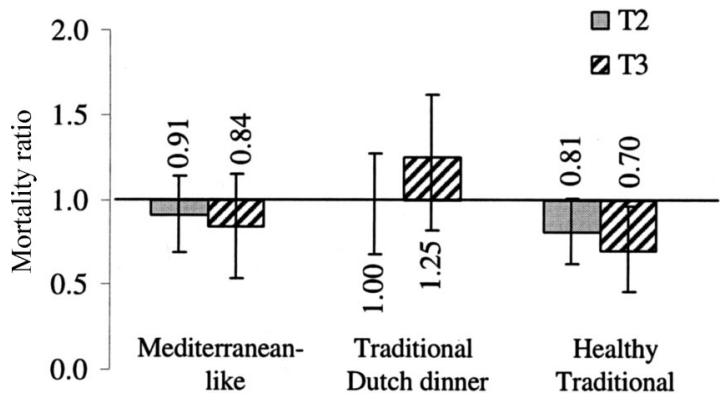

FIGURE 1. Mortality ratios of Cox proportional hazards regression for tertiles of the 3 major principal components for women $(n=5427)$ of the Dutch European Prospective Investigation into Cancer and Nutrition-Elderly cohort adjusted for age, cigarette smoking, education level, diabetes, energy intake, BMI, waist-to-hip ratio, physical activity at work, and physical activity during leisure time. Tertile (T) 1 was the reference category. could be studied, and it was only possible to detect strong associations. By excluding prevalent cases of cardiovascular disease or cancer and deaths that occurred during the first year of followup, the results are unlikely to be biased by present diseases.

It is of interest whether the discerned patterns are also observed in other populations. When findings from various studies are compared, the methodologic choices of the studies may influence factor solutions (5). Nevertheless, analogous dietary patterns have been identified in several studies and labeled either "prudent" or "healthy", often in combination with a "Western" dietary pattern (16-20). Our Healthy Traditional dietary pattern corresponds appreciably with the prudent and healthy dietary patterns, which are represented by higher intakes of vegetables, fruit, and whole grains and also often by higher intakes of dairy products, fish, or poultry. Adherence to these patterns was shown to lower coronary heart disease risk (19, 21), stroke risk (17), and mortality risk (18).

Red and processed meats are important contributors to the Western dietary patterns identified in the various studies, often in combination with intake of dairy products, refined grains, and sweets. Our Traditional Dutch dinner dietary pattern is also characterized by a higher consumption of meat, but other aspects are 
less comparable to the Western dietary patterns identified in other studies (16).

Because consumption patterns are culturally determined, we compared our findings with results from patterning studies in other Dutch populations. Van Dam et al (22) extracted 3 dietary patterns from 46 energy-adjusted (ie, residual method) food groups in a large sample of Dutch men and women from the MORGEN study. In addition, Balder et al (23) reported 5 different dietary patterns for Dutch women from the Netherlands Cohort Study on Diet and Cancer. Despite some methodologic differences, our Mediterranean-like dietary pattern showed strong similarities with the first patterns extracted by van Dam et al and Balder et al. The second pattern of van Dam et al was similar to our Traditional Dutch dinner dietary pattern - high in meat and alcoholic beverage (ie, beer) intakes and low in intakes of dairy products, cakes, and added sugar. Our Healthy Traditional dietary pattern, which is similar to the reported prudent dietary patterns, was not, surprisingly, found in these 2 Dutch studies. A possible explanation may be that participants in the other cohorts were younger, which would suggest that this Healthy Traditional dietary pattern is disappearing.

The Mediterranean diet has gained considerable attention for its reported positive health effects (24-26). In one study conducted in elderly European persons, a reduced mortality risk of $23 \%$ was reported for adherence to the Mediterranean diet (27), whereas no statistically significant association was found in another study (28). Recently, Trichopoulou et al (29) reported that the Mediterranean Diet Score was associated with an increased survival in the EPIC-Elderly study, which combined 9 European countries (of which the present cohort is a subcohort). However, this association was not significant for 6 of these 9 countries, and the association was absent for the Netherlands, Germany, and Italy (29).

Nutritional habits differ substantially between countries and, therefore, dietary patterns that can be found in Mediterranean countries may inadequately assess the diet in Western European populations. We labeled the first component in our study Mediterranean-like, but it may, in fact, not correspond well with the traditional Mediterranean diet and may not produce the same potential beneficial effects on health and longevity. For example, although vegetable oil consumption increased from the first to the third tertile, it remained low. This is illustrated by the intake of monounsaturated fatty acids, which even decreased along tertiles of the pattern. Wine, fish, and vegetable intakes also remained low.

In the 9 countries of the EPIC-Elderly study, 2 major dietary patterns were identified from the same 22 food groups we used (6). The first was a vegetable-based dietary pattern that was high in intakes of vegetable oils, fruits, pasta, rice and other cereals, vegetables, and legumes. The second pattern was the sweet- and fat-dominated diet and was high in intakes of nonalcoholic beverages, potatoes, and margarine. Neither of these 2 patterns showed substantial similarity with any of the 3 patterns resulting from our analyses. In the international study, the Netherlands was not associated with the sweet- and fat-dominated dietary pattern, whereas Dutch participants scored slightly negative on the vegetable-based dietary pattern, which indicated that a larger part of the Dutch participants showed an eating pattern opposite to this component. In a subsequent study by Bamia et al (Bamia C, Trichopolous D, Ferrari P, et al, unpublished observations, 2006), a strong association of the vegetable-based pattern with overall mortality was observed for the Netherlands. These results suggest a possible health benefit of a vegetable-based diet as identified by Bamia et al. However, although insight into prevailing eating patterns and their occurrence throughout Europe is interesting, the patterns resulting from this international study do not teach us much about Dutch dietary practice. Our results may have stronger implications for elderly Dutch persons.

Despite the small number of deaths in our study, we found a significant association between adherence to a Healthy Traditional Dutch dietary pattern and overall mortality-mortality risk was reduced $30 \%$ for the highest compared with the lowest tertile. Similar effects have been found for prudent dietary patterns identified in other populations, patterns that are comparable to our Healthy Traditional pattern. A traditional Dutch meal consists of a combination of meat, potatoes, and cooked vegetables, although the observed mortality reduction cannot be explained by meat consumption. In addition, consumption of milk and dairy products has traditionally been high and fruit consumption has been promoted for many years in the Netherlands. Even though consumption patterns have been changing in recent years (30), this traditional pattern appears to still exist in older women. Additional research conducted in younger women is needed to discern whether this pattern is disappearing. Some adaptations to a more modern variant of the Healthy Traditional dietary pattern (eg, with increased fish consumption and moderate alcohol consumption) may even improve its composition.

In conclusion, our findings showed that a healthy dietary pattern variant can be distinguished within the traditional Dutch diet. In this population of older Dutch women, a healthy traditional Dutch diet, rather than a Mediterranean diet, increases longevity. Because the Healthy Traditional dietary pattern fits in well with Dutch consumption habits, adherence to such a diet seems feasible and may be encouraged.

HBBM, MCO, PHMP, CB, and NS contributed to the design of the study and the data collection. $\mathrm{PMCMW}, \mathrm{MCO}$, and $\mathrm{CTMvR}$ were responsible for the statistical analyses of the data. PMCMW drafted the manuscript, which was critically revised by all authors. None of the authors had a conflict of interest.

\section{REFERENCES}

1. World Health Organization. Diet, nutrition and the prevention of chronic diseases. World Health Org Tech Rep Ser 2003;916:1-149.

2. de Groot LC, Verheijden MW, de Henauw S, Schroll M, van Staveren WA. Lifestyle, nutritional status, health, and mortality in elderly people across Europe: a review of the longitudinal results of the SENECA Study. J Gerontol A Biol Sci Med Sci 2004;59:1277-84.

3. World Health Orgaznization. Ageing: exploding the myths. Geneva, Switzerland: World Health Organization, 1999.

4. Hu FB. Dietary pattern analysis: a new direction in nutritional epidemiology. Curr Opin Lipidol 2002;13:3-9.

5. Newby PK, Tucker KL. Empirically derived eating patterns using factor or cluster analysis: a review. Nutr Rev 2004;62:177-203.

6. Bamia C, Orfanos P, Ferrari P, et al. Dietary patterns among older Europeans. Br J Nutr 2005;94:100-13.

7. Slimani N, Kaaks R, Ferrari P, et al. European Prospective Investigation into Cancer and Nutrition (EPIC) calibration study: rationale, design and population characteristics. Public Health Nutr 2002;5:1125-45.

8. Riboli E, Hunt KJ, Slimani N, et al. European Prospective Investigation into Cancer and Nutrition (EPIC): study populations and data collection. Public Health Nutr 2002;5:1113-24.

9. Boker LK, van Noord PA, van der Schouw YT, et al. Prospect-EPIC Utrecht: study design and characteristics of the cohort population. European Prospective Investigation into Cancer and Nutrition. Eur J Epidemiol 2001;17:1047-53. 
10. Blokstra A, Seidell JC, Smit HA, Bueno-de-Mesquita HB, Verschuren WMM. The Monitoring Project on Risk Factors for Chronic Diseases (MORGEN project) annual report 1997 (in Dutch). Bilthoven, Netherlands: RIVM (National Institute for Public Health and the Environment), 1998.

11. Ocke MC, Bueno-de-Mesquita HB, Goddijn HE, et al. The Dutch EPIC food frequency questionnaire. I. Description of the questionnaire, and relative validity and reproducibility for food groups. Int J Epidemiol 1997;26(suppl):S37-48.

12. Ocke MC, Bueno-de-Mesquita HB, Pols MA, Smit HA, Staveren WAv, Kromhout D. The Dutch EPIC food frequency questionnaire. II. Relative validity and reproducibility for nutrients. Int J Epidemiol 1997;26(suppl):S49-58.

13. Voedingscentrum. Dutch food consumption table 1996. Den Haag, Netherlands: Voedingscentrum, 1996.

14. Willett W. Nutritional Epidemiology. New York, NY: Oxford University Press, 1998.

15. Chatfield C, Collins AJ. Introduction to multivariate analysis. In: Chatfield C, Zidek JV, eds. London, United Kingdom: Chapman and Hall, 1995.

16. Terry P, Hu FB, Hansen H, Wolk A. Prospective study of major dietary patterns and colorectal cancer risk in women. Am J Epidemiol 2001; 154:1143-9.

17. Fung TT, Stampfer MJ, Manson JE, Rexrode KM, Willett WC, Hu FB. Prospective study of major dietary patterns and stroke risk in women. Stroke 2004;35:2014-9.

18. Osler M, Heitmann BL, Gerdes LU, Jorgensen LM, Schroll M. Dietary patterns and mortality in Danish men and women: a prospective observational study. Br J Nutr 2001;85:219-25.

19. Hu FB, Rimm EB, Stampfer MJ, Ascherio A, Spiegelman D, Willett WC. Prospective study of major dietary patterns and risk of coronary heart disease in men. Am J Clin Nutr 2000;72:912-21.

20. Kerver JM, Yang EJ, Bianchi L, Song WO. Dietary patterns associated with risk factors for cardiovascular disease in healthy US adults. Am J Clin Nutr 2003;78:1103-10.

21. Fung TT, Willett WC, Stampfer MJ, Manson JE, Hu FB. Dietary patterns and the risk of coronary heart disease in women. Arch Intern Med 2001;161:1857-62.

22. van Dam RM, Grievink L, Ocke MC, Feskens EJ. Patterns of food consumption and risk factors for cardiovascular disease in the general Dutch population. Am J Clin Nutr 2003;77:1156-63.

23. Balder HF, Van den Brandt PA, Goldbohm RA. Dietary patterns and cancer risk in a prospective cohort study. In: Balder HF. Diet and cancer. The explanatory value of dietary patterns. PhD dissertation. Göttingen, Netherlands: Cuvillier Verlag, 2004.

24. Trichopoulou A, Kouris-Blazos A, Wahlqvist ML, et al. Diet and overall survival in elderly people. BMJ 1995;311:1457-60.

25. Osler M, Schroll M. Diet and mortality in a cohort of elderly people in a north European community. Int J Epidemiol 1997;26:155-9.

26. Trichopoulou A, Costacou T, Bamia C, Trichopoulos D. Adherence to a Mediterranean diet and survival in a Greek population. N Engl J Med 2003;348:2599-608.

27. Knoops KT, de Groot LC, Kromhout D, et al. Mediterranean diet, lifestyle factors, and 10-year mortality in elderly European men and women: the HALE project. JAMA 2004;292:1433-9.

28. Haveman-Nies A, de Groot LP, Burema J, Cruz JA, Osler M, van Staveren WA. Dietary quality and lifestyle factors in relation to 10-year mortality in older Europeans: the SENECA study. Am J Epidemiol 2002;156:962-8.

29. Trichopoulou A, Orfanos P, Norat T, et al. Modified Mediterranean diet and survival: EPIC-elderly prospective cohort study. BMJ 2005;330: 991.

30. Health Council of the Netherlands. Significant trends in food consumption in the Netherlands. The Hague, Netherlands: Health Council of the Netherlands, 2002. 